\title{
Priapismo maligno: un caso manejado de forma conservadora
}

\section{Malignant priapism: conservative management of a case}

\author{
A. Tienza, I. Merino, J.M. Velis, R. Algarra, M.D. Hernández, J.E. Robles
}

\section{RESUMEN}

El priapismo es una urgencia urológica que requiere especial valoración, especialmente en la diferenciación de priapismo isquémico y no isquémico. El manejo inicial es mediante aspiración y gasometría de la sangre de los cuerpos cavernosos. Presentamos un paciente de 69 años diagnosticado de carcinoma de urotelio vesical T2, con metástasis en uretra/cuerpo cavernoso que acude a urgencias por edema y tumefacción peneano de varios días de evolución. Ante el pronóstico malo y las pruebas de imagen realizadas se decide tratamiento de soporte y quimioterápico.

Palabras clave. Priapismo maligno. Carcinoma urotelio. Adenocarcinoma de próstata.

\begin{abstract}
Priapism is an urological emergency which requires investigation, especially to differentiate between ischemic and non-ischemic priapism. Initial management is carried out through aspiration and gasometry of blood from the corpus cavernosum. We report the case of a 69-year-old patient with urothelium carcinoma of the bladder T2 G3 and metastasis in urethra/corpus cavernosum who requested an emergency consultation because of edema and a penile erection lasting several days. Due to the poor prognosis and the imaging test, a conservative management was carried out.
\end{abstract}

Key words. Malignant priapism. Urothelial carcinoma. Prostate adenocarcinoma.
Departamento de Urología. Clínica Universidad de Navarra

Recepción: 26 de junio de 2012

Aceptación provisional: 28 de septiembre de 2012

Aceptación definitiva: 15 de octubre de 2012

\section{Correspondencia:}

A. Tienza

Departamento de Urología

Clínica Universidad de Navarra

Avda. Pío XII, 36

31008 Pamplona

E-mail: atienza@unav.es 


\section{INTRODUCCIÓN}

El priapismo es una urgencia urológica, cuyo término viene del dios de la antigua Grecia, Priapus, hijo de Afrodita y Zeus ${ }^{1}$.

La definición más usada de priapismo es una erección prolongada, normalmente superior a 4-6 horas de duración, sin estimulación sexual.

Lo más importante ante un caso de priapismo es diferenciar el tipo, alto o bajo flujo, ya que el manejo es distinto.

Presentamos un caso de priapismo maligno debido a un carcinoma vesical infiltrante con metástasis en uretra/cuerpo cavernoso.

\section{CASO CLÍNICO}

Varón de 69 años que acudió en mayo de 2011 al servicio de Urgencias presentando edema peneano de 4 días de evolución y erección no completa de más de 24 horas, hemoglobina en sangre de $8,2 \mathrm{mg} / \mathrm{dl}$ y 140 hematíes por campo en el análisis de orina.

Entre sus antecedentes personales se encuentra en 2001 un carcinoma vesical de urotelio T2b, G3 diagnosticado mediante RTU vesical y tratado mediante 6 ciclos de quimioterapia consistente en taxol $245 \mathrm{mg}$, cisplatino $136 \mathrm{mg}$, gemcitabina $1.456 \mathrm{mg}$ y radioterapia externa 64Gy a mitad de ciclos que concluyó con remisión completa hasta 2005. Los años sucesivos presenta recidivas de tumores vesicales superficiales que se electrocoagulan. En el año 2008 presentó elevación del PSA de $5 \mathrm{ng} / \mathrm{ml}$ realizando biopsia de próstata informada como adenocarcinoma de próstata $3+3$, Gleason 6. Se trató mediante braquiterapia intersticial con semillas de Iodo $^{125}$ en 2009.

A finales de 2010 refirió molestias premiccionales observando mediante cistoscopia lesión en uretra prostática. Se realizó RTU-biópsica que fue informada como infiltración de carcinoma de urotelio con diferenciación escamosa. La lesión fue tratada mediante resección y 6 ciclos de quimioterapia consistente en gemcitabina $3.300 \mathrm{mg}$ $\left(1.750 \mathrm{mg} / \mathrm{m}^{2}\right)$, cisplatino $94 \mathrm{mg}\left(50 \mathrm{mg} / \mathrm{m}^{2}\right)$ finalizado en marzo de 2011.

En revisión de abril de 2012 continuaba con disminución del PSA hasta valor de $0,6 \mathrm{ng} / \mathrm{ml}$; la cistoscopia mostraba un proceso inflamatoriocicatricial a nivel de uretra y en la tomografía por emisión de positrones (PET/CT). Se veían varias imágenes hipermetabólicas, sugestivas de enfermedad en pared lateral derecha e izquierda de vejiga, infiltración de vesícula seminal y depósito en uretra peneana englobando cuerpo caver- noso izquierdo, así como afectación ganglionar (Fig. 1 parte inferior. Fig. 2, mitad derecha). Se le recomendó tratamiento quimioterápico que no llegó a recibir.

Revisada su historia clínica en el servicio de Urgencias se decidió el ingreso. Se realizó tratamiento sintomático, transfusión de hemoderivados, tomografía axial computerizada de abdomen (TAC) (Fig. 1 parte superior. Fig. 2, mitad izquierda) para re-estadificación y tratamiento quimioterápico de segunda línea mediante vinflunina bitartrato $500 \mathrm{mg}(280 \mathrm{mg} / \mathrm{m} 2)$ dosis única.

El edema y la erección desaparecieron y recibió el alta tras cinco días de ingreso. El paciente falleció 2 meses después.

\section{DISCUSIÓN}

El priapismo supone una urgencia de múltiples causas en los servicio de Urología. Una de las causas es la maligna correspondiendo al 3-8\% de todos los priapismos $^{2,3}$. Vilke y col encontraron una incidencia global de priapismo de 1,5 por 100.000 personas-año ${ }^{4,5}$, y de 2,9 en mayores de 40 años ${ }^{5}$.

Esta patología es extremadamente rara, causada por extensión directa o metástasis en pene de tumores, hasta un $75 \%$ de origen genitourinario ${ }^{2,4,6}$. El carcinoma de urotelio en vejiga es el responsable del $27-35 \%$, seguido por el adenocarcinoma de próstata con 26-32\%, pudiendo ser causa los tumores renales, o incluso los de origen digestivo ${ }^{7,8}$.

Un $12 \%$ cursa sin síntomas, por lo que supone una patología infraestimada. Solo un $30-40 \%$ de la metástasis en pene provocan priapismo ${ }^{7}$.

La clínica de aparición es una tumefacción peneana, más o menos duradera, recurrente o no, y pudiendo ser o no dolorosa.

El tipo de priapismo es crucial. Si el origen es maligno lo habitual es que corresponda a bajo flujo o isquémico, estando también descrito de alto flujo ${ }^{3,5}$. El priapismo de bajo flujo o isquémico se debe a oclusión venosa y está muy relacionado con medicación vasoactiva intracavernomatosa. La erección es completa y dolorosa. El diagnóstico se hace mediante aspiración de sangre intracavernomatosa y gasometría, mostrando valores de $\mathrm{pH}$ $<7,25$, oxígeno $<30$ mm Hg y dióxido de car- 


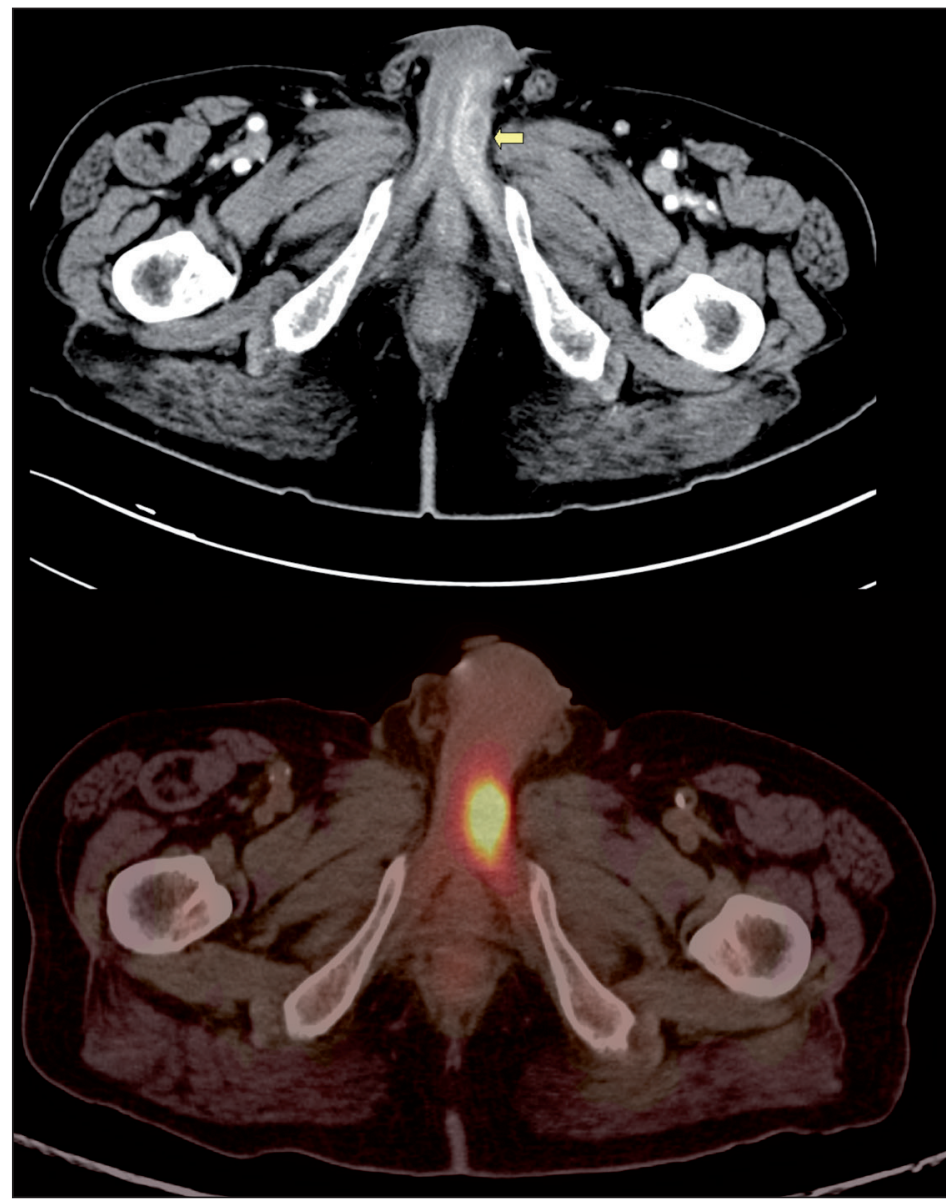

Figura 1. Corte axial de TAC de abdomen del ingreso tras acudir a urgencias, la flecha señala cuerpo cavernoso afectado. Imagen equivalente en el PET/TC realizado un mes antes con captación hipermetabólica.

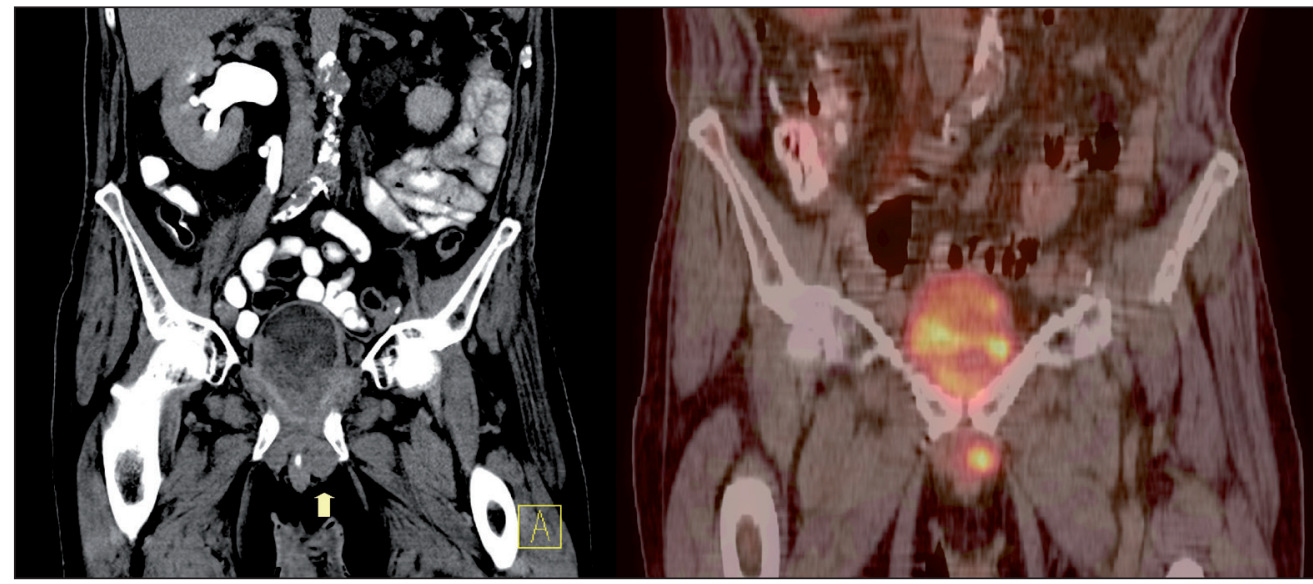

Figura 2. Corte coronal de TAC de abdomen de ingreso tras acudir a urgencias objetivando lesiones en vejiga, la flecha señala cuerpo cavernoso afectado. Imagen equivalente en el PET/TC realizado un mes antes con los tres focos hipercaptantes. 
bono $>60 \mathrm{~mm} \mathrm{Hg}{ }^{1,6}$. Requiere actitud urgente y aspirado, lavado o infusión de medicación en cuerpos cavernosos.

Por otro lado, el priapismo de alto flujo o no isquémico provoca erecciones no dolorosas y el aspecto de la sangre es rojo con oxígeno elevado ${ }^{6}$. Puede deberse a traumatismo peneano, perineal o pélvico, o incluso a neoplasia y conviene descartar la enfermedad de Fabry ${ }^{3,5}$.

El priapismo de alto flujo no supone una urgencia y no supone un riesgo de daño nervisoso y posible disfunción eréctil residual.

También está descrito el priapismo recurrente, presente en enfermedades hematológicas.

En nuestro caso se decidió una actitud conservadora sin aspiración ni lavados intracavernomatosos debido a una evolución prolongada, una erección no completa y no dolorosa, el antecedente de braquiterapia y el pronótico malo. A día de hoy, en casos concretos se podría apostar por un manejo no invasivo, evitando lavados y biopsia de cuerpo cavernoso pero requiriendo pruebas de imagen. Son útiles la ecografía doppler, la tomografía axial o la resonancia magnética tanto para el diagnóstico ${ }^{4}$ como para la extensión de la enfermedad ${ }^{3,9}$.

Además en los casos de priapismo maligno, debería hacerse una biopsia del cuerpo cavernoso ${ }^{4,5,7,8}$. En nuestro departamento se acepta el uso del PET/CT.

Este tipo de manejo también podría respaldarse por el pronóstico nefasto ${ }^{3,4,7,8}$. Si la enfermedad está muy avanzada, el $80 \%$ fallece en los primeros 6 meses $^{9}$ con una media de supervivencia de 47 semanas $^{4}$. Por esta razón la mayoría de los pacientes solo requieren tratamiento de soporte o paliativo ${ }^{4}$.

En casos de origen maligno localizado, la cirugía mediante penectomía podría estar justificada, pero el tratamiento principal sería la quimioterapia. En casos avanzados de patología tumoral con origen genitourinario, la quimioterapia de segunda línea mediante vinflunina puede ser un alivio para el paciente ${ }^{10}$.

El priapismo suele ser una urgencia urológica. Si transcurre demasiado tiempo se produce daño nervioso que provocará disfunción eréctil.

El priapismo de origen maligno tradicionalmente manejado mediante aspiración y lavados intracavernomatosos, biopsia de la lesión y quimioterapia con o sin cirugía, puede ser sustituido mediante pruebas de imagen y administración de nuevos fármacos quimioterápicos, debido al pronóstico pobre.

\section{BIBLIOGRAFÍA}

1. Cherian J, Rao AR, Thwaini A, Kapasi F, Shergill IS, SAmman R. Medical and surgical management of priapism. Postgrad Med J 2006 82: 89-94.

2. Celma Doménech A, Planas Morin J, de Torres Ramírez I, Bestard Vallejo J, Mir Marisma C, Morote Robles J. Priapismo secundario a infiltración peneana por contigüidad de carcinoma vesical infiltrante. Actas Urol Esp 2008; 32: 749-751.

3. Eguíluz Lumbreras P, Palacios Hernández A, Heredero Zorzo O, Cañada de Arriba F, García García J, Gómez ZancAJo VR et al. Malignant priapism and secondary bladder cancer. Arch Esp Urol 2009; 62: 239-242.

4. Cherian J, Rajan S, Thwaini A, Elmasry Y, Shah T, PURI R. Secondary penile tumours revisited. Int Semin Surg Oncol 2006; 11; 3: 33.

5. Vilke GM, Harrigan RA, Ufberg JW, Chan TC. Emergency evaluation and treatment of priapism. J Emerg Med 2004; 26: 325-329.

6. Harmon W, Nehra A. Priapism: Diagnosis and management. Mayo Clinic Proc 1997; 72: 350355.

7. Morga Egea JP, Ferrero Doria R, Guzmán Martínez-Valls PL, Navas Pastor J, García Ligero J, García García F et al. Priapismo Metastátisco. Presentación de cuatro nuevos casos y revisión de la literatura. Arch Esp Urol 2000; 53; 447-452.

8. Chan PT, Bégin LR, Arnold D, Jacobson SA, Corcos J, BRock GB. Priapism secondary to penile metastasis: A report of two cases and a review of the literature. J Surg Oncol 1998; 68: 51-59.

9. SÖnmez NC, CoŞKun B, ArISAn S, Güney S, DalKILIÇ A. Early penile metastasis from primary bladder cancer as the first systemic manifestation: a case report. Cases J 2009; 14; 2: 7281.

10. Stenzl A, Cowan NC, De Santis M, Kuczyk MA, Merseburger AS, Ribal MJ et al. Treatment of Muscle-Invasive and Metastatic Bladder Cancer: Update of the EAU Guidelines. Actas Urol Esp 2012; 36; 449-460. 